\title{
Las Tecnologías de la Información y la Comunicación como vehículo de innovación y emprendimiento en el Espacio Europeo de Educación Superior: una propuesta docente basada en la comunicación digital
}

\author{
Sergio Álvarez García \\ Universidad Rey Juan Carlos (URJC) \\ sergio.alvarez@urjc.es
}

Recibido: $19 / 11 / 2012$

Aceptado: 23/01/2013

\begin{abstract}
Resumen
Este artículo describe un modelo de explotación de la comunicación y de la tecnología orientado a la consecución de objetivos didácticos, profesionales e institucionales en un contexto determinado: las aulas de la Facultad de Ciencias de la Comunicación de la Universidad Rey Juan Carlos. Este modelo gira en torno a la configuración de un entorno de trabajo profesional orientado por objetivos y proyectos reales que cubren necesidades de la propia institución en la que se imparte la docencia y que actúa como cliente. En lo didáctico, se potencia el aprendizaje autónomo del estudiante mediante estrategias metodológicas activas, que resultan óptimas, además, para sentar las bases necesarias de una actitud y de una aptitud de emprendimiento.

Palabras clave: Comunicación multimedia, Innovación, Docencia, Universidad, Emprendimiento

\section{Information and Communications Technologies as a Vehicle for Innovation and Entrepreneurship in the European Higher Education Area: a Teaching Proposal Based on Digital Communication}

\begin{abstract}
This paper describes an operational model of communication and technology aimed at achieving educational, professional and institutional objectives in context: the classrooms of the Faculty of Communication Sciences at the Universidad Rey Juan Carlos. This model focuses on setting up a professional working environment and goal-oriented projects covering actual needs of the institution in which teaching is taught and acts as a client. In teaching, autonomous learning is enhanced by active methodological strategies that are optimal also to lay the foundations of an attitude and an aptitude for entrepreneurship.

Keywords: Multimedia Communication, Innovation, Teaching, University, Entrepreneurship

\section{Referencia normalizada}

ÁLVAREZ GARCÍA, Sergio (2013): "Las Tecnologías de la Información y la Comunicación como vehículo de innovación y emprendimiento en el Espacio Europeo de Educación Superior: una propuesta docente basada en la comunicación digital”. Estudios sobre el Mensaje Periodístico. Vol. 19, Núm. especial abril, págs.: 583-592. Madrid, Servicio de Publicaciones de la Universidad Complutense.

Sumario: 1. Introducción; 1.1. Justificación y límites; 1.2. Objetivos; 1.2.1. Objetivos pedagógicos y didácticos; 1.2.2. Objetivos técnicos; 1.2.3. Objetivos institucionales. 2. Metodología; 2.1. Investigación; 2.2. Innovación. 3. Desarrollo; 3.1. Recursos; 3.1.1. Web CT (Campus Virtual de la URJC); 3.1.2. Joomla; 3.1.3. Wordpress; 3.2. Actividades; 3.3. Productos de comunicación. 4. Conclusiones; 4.1. Obstáculos; 4.2. Beneficios. 5. Referencias bibliográficas.
\end{abstract}




\section{Introducción}

El modelo de Grado propuesto en el marco del Espacio Europeo de Educación Superior (EEES) toma como criterio fundamental para la organización didáctica la carga de trabajo del estudiante (en su denominación original en inglés, "Student'sWorkload") y, como señala el Proyecto TUNING, pone el acento en una Enseñanza Superior que busca la formación de sujetos humanistas, cooperativos, investigadores y reflexivos. El uso de estrategias docentes basadas en el aprendizaje cooperativo y el aprendizaje autónomo del estudiante sigue ese camino, demandado por la sociedad actual a nuestros estudiantes y que con el foco situado en lograr "mayores niveles de empleabilidad y ciudadanía" (Pulido, 2003: 1) pretende construir una universidad más crítica y constructiva de la realidad social actual.

La Declaración de Bolonia tiene como una de sus principales consecuencias el inicio de procesos de transformación de los sistemas educativos de enseñanza superior de la mayoría de los países europeos. Para las universidades, las reformas que supone el llamado "Plan Bolonia" motivan otro análisis: "la sintonización en términos de estructuras y programas y de la enseñanza propiamente dicha", en la que desempeñan un "importante papel, además de los objetivos que fije la colectividad académica, los perfiles académicos y profesionales que exige la sociedad", además del "esclarecimiento del nivel de formación que debe lograrse en términos de competencias y resultados del aprendizaje." (González y Wagenaar, 2003: 26).

La "Estrategia 2020" (Comisión Europea, 2012: web) se orienta desde su lema a un "crecimiento inteligente, sostenible e integrador" que tiene como motor fundamental el emprendimiento, el "movimiento" de una sociedad a través de sistemas educativos que faciliten la entrada de los jóvenes en el mercado de trabajo.

Este conjunto de orientaciones, reformas y objetivos marca algunos de los rumbos del docente actual, obligado a impulsar cambios en las metodologías didácticas, a adaptarse a un contexto en el que se producen modificaciones sustantivas en los roles del profesorado y del alumnado, en las condiciones espacio-temporales del proceso de enseñanza-aprendizaje, y en los instrumentos que facilitan y potencian la búsqueda, análisis y selección de la información, $\mathrm{y}$, en consecuencia, la adquisición de conocimiento.

El escenario de la comunicación -como sector profesional, como objeto de investigación, como contenido formativo, como Ciencia- no sólo no es indiferente a estos factores, sino que los acumula y expresa con intensidad a través de uno de sus instrumentos más propios: las Tecnologías de la Información y la Comunicación.

La comunicación multimedia, latente en nuevos soportes como la Prensa Digital, causa y efecto de nuevos formatos -prensa gratuita, medios de comunicación interactivos- y expresiones innovadoras del periodismo -con el "periodismo ciudadano" como máximo exponente- ha cobrado una notoriedad económica en el mercado de la Comunicación. Y esta notoriedad se traslada al aula universitaria: "Los nuevos Grados en Comunicación que van apareciendo al amparo del espíritu de Bolonia están siendo diseñados desde una perspectiva relacionada con la incidencia de la industria de la Comunicación en los ámbitos sociales, económicos y académicos" (Ortiz Sobrino, 2009: 38). 


\subsection{Justificación y límites}

Es preciso aclarar que este proyecto de innovación educativa no concentra su valor en la naturaleza del cambio metodológico que supone, sino en la forma en que se contextualiza, instrumentaliza y evalúa el trabajo que realizan los alumnos para alcanzar los objetivos didácticos de sus respectivas asignaturas.A través de la configuración de un entorno profesional que plantea problemas y objetivos reales y específicos para cada asignatura y grupo de alumnos, el docente adapta las demandas de un cliente real a las estrategias que recoge la guía didáctica en lo que a actividades se refiere.

Las Tecnologías de la Información y la Comunicación son instrumentos esenciales en todo este proceso, pues permiten economizar esfuerzos y recursos en la consecución de resultados profesionales, motivan a los estudiantes al emprendimiento, y sirven de vehículo al desarrollo de tareas de enseñanza-aprendizaje que se adaptan e integran de forma natural en ese entorno profesional que se configura. La instrumentalización de la actividad que se genera en ese entorno suma el elemento de la tecnología emergente a la caracterización del proyecto como innovador.

La última etapa del acto didáctico coincide con el cierre del proyecto: la evaluación de los resultados del trabajo del alumnado y el seguimiento de su actividad con su posterior calificación coinciden como fruto de una configuración profesional del entorno de enseñanza-aprendizaje. Pero además, de esta convivencia surge un valor añadido de innovación, pues en el proceso de evaluación participan los compañeros (pares) y agentes externos a la asignatura. El cliente real, cuyas demandas dieron sentido al proyecto y lo iniciaron en la práctica, interviene ahora valorando en qué medida los resultados del trabajo de los estudiantes responde a lo solicitado. Los alumnos del grupo-clase, mediante un cuestionario que les obliga a profundizar en lo realizado por otros grupos de compañeros, analizan los resultados y los comentan. El profesor reserva un porcentaje importante de la evaluación y de la calificación a estas valoraciones y comentarios, garantizando así la corrección en la configuración del entorno de trabajo y cerrando el proyecto con una conexión definitiva entre el acto didáctico, la práctica profesional y la reflexión y crítica exigida al estudiante universitario.

\subsection{Objetivos}

La propuesta de este proyecto de innovación docente recoge una serie de objetivos que se orientan a responder a los valores que justifican su diseño y puesta en marcha. Son los siguientes:

\subsubsection{Objetivos pedagógicos y didácticos}

» Ofrecer a los estudiantes un entorno de trabajo real para un proceso activo de aprendizaje de los contenidos curriculares (conceptuales, procedimentales y de actitud) rico, diverso, flexible, extensible, actualizado y de calidad, que les permita comprender, en su globalidad, el desempeño profesional de la planificación, el diseño, el desarrollo y la integración de campañas, acciones y productos de comunicación multimedia orientados al cumplimento de necesidades y objetivos determinados de comunicación integral.

» Poner a disposición del alumnado objetos y actividades de aprendizaje con un nivel de granularidad suficiente para garantizar un aprendizaje adaptativo, de ma- 
nera que los estudiantes que cursen las diferentes asignaturas implicadas en el proyecto puedan adaptar su ritmo de aprendizaje a sus particularidades y necesidades educativas.

» Facilitar las capacidades de los alumnos para la compresión, análisis, síntesis, crítica y creatividad, mediante los modelos de cooperación, motivación y aprendizaje abierto del currículo que facilitan las herramientas, aplicaciones y servicios TIC propuestos.

» Ofrecer al alumnado conocimiento aplicado sobre los fundamentos, componentes, estrategias, técnicas y sistemas de comunicación integral actuales, y su implicación en la planificación, el diseño, el desarrollo y la integración de campañas, acciones y productos de comunicación multimedia.

» Proporcionar a los estudiantes los espacios, orientaciones y recursos necesarios para la creación y publicación de porfolios digitales personales, integrables en sus currículos.

\section{2. 2. Objetivos técnicos}

» Modelar un entorno de trabajo digital que emule situaciones profesionales reales de producción multimedia y planificación de medios en la Red.

» Optimizar el uso de Campus Virtual como espacio de interpretación y evaluación de los resultados obtenidos para su adaptación a las exigencias conceptuales y procedimentales de naturaleza académica y docente en relación con las asignaturas integradas en el proyecto.

» Planificar y diseñar, producir, desarrollar e integrar campañas, acciones y productos de comunicación multimedia siguiendo estándares profesionales, como los que establece, por ejemplo, la IAB (Interactive Advertising Bureau, 2012: web) para este ámbito.

» Generar contenidos de comunicación multimedia según los requisitos técnicos especificados por las publicaciones digitales de la Facultad de Ciencias de la Comunicación de la URJC que se vayan a alimentar.

» Publicar los productos de comunicación multimedia desarrollados en el marco del proyecto de forma profesional y responsable, tomando en consideración las especificaciones y normas sobre accesibilidad internacionales de la WAI (Web Accesibility Initiative, 2011: web) así como las recomendaciones ofrecidas por distintas instituciones como el CENATIC (Centro Nacional de Referencia de Aplicación de las TIC basadas en fuentes abiertas, 2012: web) o la Organización Nacional de Ciegos Españoles (Fundación ONCE, 2012: web) para facilitar el acceso y disfrute de los mismos a cualquier alumno o usuario independiente de sus características físicas, perceptivas o de edad.

» Configurar un entorno de producción multimedia que unifique y optimice las herramientas y recursos TIC utilizados en el proyecto - destacan el gestor de contenidos multimedia para el desarrollo de publicaciones Joomla, o el gestor de weblogs Wordpress para el trabajo con los porfolios personales de los estudiantes- y las conecte directamente con los espacios e instrumentos de la Facultad de Ciencias de la Comunicación de la URJC. 


\section{2. 3. Objetivos institucionales}

» Dirigir las actividades de aprendizaje hacia la toma de conciencia, por parte de todos los participantes en el proyecto, de su integración en la Universidad Rey Juan Carlos y de la necesidad de adquirir responsabilidades y cooperar en la suma de esfuerzos, ideas e iniciativas para su mejora.

» Aportar productos y soluciones profesionales para la satisfacción de necesidades y demandas globales o específicas de la Facultad de Ciencias de la Comunicación de la URJC en materia de comunicación multimedia, que no hayan podido ser cubiertas con los recursos disponibles.

\section{Metodología}

Es necesario aclarar que en este apartado conviven dos espacios metodológicos distintos: uno, el diseñado para evaluar, interpretar y extraer conclusiones del proyecto de innovación que se describe en estas líneas; y otro, el que se configura para el propio desarrollo de las tareas y procesos de dicho proyecto.

\subsection{Investigación}

En el primero de estos espacios, se combina la aplicación de tres instrumentos:

» Evaluación del trabajo del estudiante en las respectivas asignaturas, llevada a cabo por el profesor. La configuración profesional del entorno de enseñanzaaprendizaje permite valorar competencias adquiridas y resultados obtenidos desde una doble perspectiva: la didáctica y la laboral.

» Observación directa participante, del docente de la asignatura mediante el seguimiento de las prácticas y de la participación del alumnado en el proyecto.

» Cuestionarios de análisis del proyecto realizados por los estudiantes participantes, que evalúan el trabajo realizado por sus compañeros e interpretan los resultados propios atendiendo al valor y significación personales e individuales.

\subsection{Innovación}

En lo que se refiere al desarrollo del proyecto propiamente dicho, la metodología toma como referencia los resultados de las prácticas desarrolladas por los profesores participantes durante los cursos académicos precedentes con los estudiantes en sus correspondientes asignaturas. Se trata de un trabajo con formato de curso piloto, en sistema de blended learning, haciendo un uso intensivo de la plataforma Campus Virtual de la Universidad Rey Juan Carlos, en combinación con herramientas de la denominada web 2.0.

En este sentido, el profesorado participante se ha guiado por el método Syllabus (sumario), una herramienta didáctica basada en un enfoque constructivista del aprendizaje, que parte del presupuesto de que las prácticas educativas activas, que implican la intervención comprometida del alumno, y centrada en los procesos de aprendizaje, ofrece mejores resultados. Este método combina los elementos clásicos del constructivismo con las apuestas por el conocimiento social (socio-constructivismo) y el uso de las TIC.

En concreto, se sigue una estrategia metodológica que toma como objetivo prioritario el lograr que los alumnos y alumnas alcancen las competencias y capacidades bá- 
sicas para desenvolverse de forma eficaz dentro de los sistemas profesionales multimodales de información, en los ámbitos estratégico, técnico, productivo y expresivo.

Esta metodología se ha concretado en técnicas de aprendizaje situado (con la configuración de un entorno profesional real) y aprendizaje basado en proyectos (para cuyo desarrollo se ha seleccionado un conjunto de recursos accesibles, de rápida aplicación productiva y expresiva, y con altos niveles de usabilidad).

\section{Desarrollo}

Con estos objetivos y metodología, este proyecto de explotación de la comunicación y de la tecnología se desarrolla fundamentalmente en torno a dos ejes: los recursos de los que se sirve y los productos resultantes de la utilización de esas herramientas.

\subsection{Recursos}

Las Tecnologías de la Información y la Comunicación (TIC) son un factor clave del proyecto, pues permiten articular el entorno profesional de trabajo y optimizar los recursos empleados por los estudiantes en el desarrollo de sus actividades. Destacan las siguientes herramientas:

\subsubsection{Web CT (Campus Virtual de la URJC)}

El Campus Virtual de la URJC está integrado sobre el LCMS propietario WebCT de la compañía norteamericana Blackboard Inc. Ha sido el entorno natural de trabajo de los alumnos de las titulaciones online y un instrumento básico para la gestión de las asignaturas de las titulaciones presenciales. Desde la plataforma se ha realizado la unificación, centralización y distribución de los planteamientos, materiales, recursos e incluso de los resultados del proyecto, además de cumplir con las necesidades propias de la gestión de las asignaturas bajo metodologías activas como las que aquí se proponen: calendario de tareas, sistemas esenciales de comunicación, tutorización online, realización y envío de trabajos, cuestionarios, exámenes, calificación.

\subsubsection{Joomla}

Sistema de Gestión de Contenidos (Content Management System, CMS), que permite crear sitios Web y aplicaciones online de carácter profesional.

Con esta herramienta se ha diseñado, desarrollado e integrado la red de microsites sobre destinos Erasmus-Munde que conforman el especial de la publicación digital de la Facultad de Ciencias de la Comunicación Aula Sur, además de los análisis de las webs de dichas universidades en el suplemento especializado en multimedia (Pantropía)

\subsubsection{Wordpress}

Avanzada plataforma semántica de publicación personal orientada a la estética, los estándares web y la usabilidad. Sistema de gestión de weblogs gratuito. Preferentemente con este gestor de contenidos, los grupos de estudiantes de la asignatura Comunicación Multimedia en sus modalidades presencial y online, han organizado la documentación generada en la planificación de sus campañas de comunicación, los 
productos desarrollados y la presentación de los mismos, todo ello publicado en la Red como porfolios particulares y colectivos de los participantes en el proyecto.

\subsection{Actividades}

El núcleo del proyecto lo constituyen las distintas actividades diseñadas por el profesorado y realizadas por los estudiantes para los bloques prácticos de las asignaturas Planificación y Desarrollo de Proyectos en la Red (Grado de Periodismo -presencial y online-), Diseño y creación multimedia (Grado de Comunicación Audiovisual), Lenguaje y Tecnologías Audiovisuales y Comunicación Multimedia (Grado de Publicidad y Relaciones Públicas -presencial y online), todas ellas de carácter obligatorio en los planes de estudio de la Universidad Rey Juan Carlos.

Han participado en el proyecto cuatro

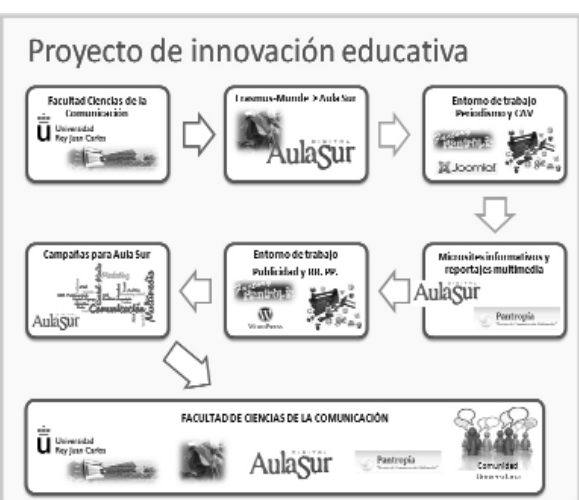

Figura 1. Esquema de desarrollo de actividades en entorno profesional real del proyecto.

Elaboración propia profesores del Departamento de Ciencias de la Comunicación II de la URJC, uno desempeñando el rol de cliente real (Jesús del Olmo, director de la revista de la Facultad de Ciencias de la Comunicación, Aulas Sur) y tres (Mario Rajas, Manuel Gértrudix y Sergio Álvarez) coordinando el trabajo de más de 400 alumnos.

Concretamente, los estudiantes han llevado a cabo las siguientes actividades profesionales, todas ellas con un producto de comunicación multimedia como resultado y como instrumento evaluable y calificable:

» Desarrollo de microsites web sobre destinos Erasmus-Munde URJC para su publicación en un especial de la revista Aula Sur de la Facultad de Ciencias de la Comunicación.

» Realización de reportajes informativos de análisis de los sitios web de las universidades del programa de movilidad URJC para su publicación en la revista del Grupo Ciberimaginario (con sede en la Facultad de Ciencias de la Comunicación de la URJC) Pantropía.

» Diseño y producción de anuncios sobre la revista Aula Sur. Bajo formato de concurso profesional publicitario, con publicación impresa y difusión real de los anuncios ganadores.

» Desarrollo de campañas de comunicación digital para la revista Aula Sur. Los grupos de estudiantes se constituyen en agencias de comunicación digital, que se hacen públicas a través de un sitio web específico. Cada agencia-grupo lleva a cabo las acciones planificadas y organiza los productos y resultados en la sección de su sitio web dedicada al porfolio, además de elaborar, publicar y exponer una presentación multimedia. 


\subsection{Productos de comunicación}

El proyecto ha respondido a las demandas profesionales iniciales mediante productos valorados como correctos y, por tanto, publicados, ejecutados o programados realmente:

» Portal web temático de pruebas con Información sobre destinos Erasmus con más de 25 minisites (http://pantropia.ciberimaginario.es/index.php/erasmus) especializados en Universidades de destino para los estudiantes de la URJC, en Europa y el resto del mundo.

» Análisis funcional de las secciones web de estas Universidades destinadas a ofrecer información a los futuros estudiantes Erasmus para evaluar las mismas, publicados con formato de reportaje multimedia en la revista Pantropía.

» Diseño, producción y competición en concurso 17 de anuncios impresos y banners digitales para la revista Aula Sur. Difusión real de los trabajos ganadores.

»Campañas de comunicación digital para la revista Aula Sur (http://delicious.com/tag/aulasur): creatividad y gestión de publicidad display; push marketing a través de e-mail y mensajería móvil; digital signage (marketing digital dinámico: pantallas multimedia interactivas exteriores o en punto de venta); campañas de publicidad cross-media; creación de sitios web de campañas de imagen de marca; acciones de comunicación viral basada en piezas de ficción; marketing de eventos con departamento de comunicación viral; desarrollo de aplicaciones (apps) para smartphones y tabletas; captación de consumidores a través de redes sociales para las relaciones personales y el entretenimiento (Facebook y Tuenti); campañas de imagen de marca a través de redes sociales para el intercambio y las relaciones profesionales (Twitter y Linkedin); consultoría de posicionamiento web (SEO); consultoría especializada en analítica web a través de Google Analytics.

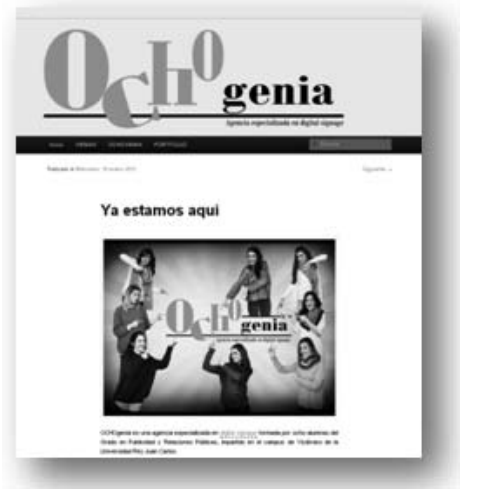

Figura 2. Portada del sitio web del grupo de estudiantes "Ochogenia" de la asignatura de Comunicación Multimedia (Ciencias de la Comunicación, URJC Vicálvaro), desarrollado con Joomla.

[Captura de pantalla]

\section{Concusiones}

A partir de lo aportado por los procesos de evaluación, análisis e interpretación diseñados para el proyecto, se puede establecer una primera conclusión, a modo de premisa y de carácter metodológico: los distintos tipos de resultados se acumulan y confunden, en una integración natural que constituye un beneficio de partida y que no excluye el aislamiento de sus elementos para responder a necesidades específicas, como la calificación académica o la valoración profesional del producto de comunicación particular.

El resto de conclusiones puede agruparse en obstáculos encontrados y beneficios obtenidos. 


\subsection{Obstáculos}

En este sentido, destaca la incidencia negativa de tres factores:

» La masificación de las aulas, que complica el desarrollo del proyecto — su configuración, el trabajo de los alumnos, su seguimiento y su evaluación - con la participación de un excesivo número de estudiantes.

» La 'tecnofobias' de algunos alumnos, que retrasan el trabajo inicial del proyecto y constituyen en ocasiones barreras a la creatividad y a la concreción de su potencial profesional.

» Las 'tecnofilias' de otros alumnos, que dirigen parte de los esfuerzos de los grupos de trabajo hacia tareas superficiales del proyecto, impidiendo así la concentración en los procesos profesionales clave y en las actividades realmente significativas para la adquisición de competencias.

\subsection{Beneficios}

En cuanto a lo desarrollado en sí, la modelización de un entorno de trabajo profesional; el uso intensivo de las TIC; la integración de instrumentos en un sistema de evaluación sumativo y formativo; el análisis y valoración del trabajo del estudiante mediante herramientas profesionales; la integración de las actividades docentes y de aprendizaje de diferentes grupos, titulaciones y modalidades en un proyecto conjunto y coordinado; la creación de productos de comunicación multimedia de diversa naturaleza (contenidos periodísticos, narraciones audiovisuales, acciones y formatos publicitarios o de comunicación corporativa) que alimentan las publicaciones digitales de la Facultad de Ciencias de la Comunicación de la URJC y satisfacen demandas de difusión o información... han generado los siguientes beneficios:

» Formativos. Se produce un aprendizaje situado y significativo para el alumnado por su carácter profesional y por la expansión -y en algunos casos configuración inicial- del e-porfolio personal. Se garantiza la adaptación de las actividades y productos a las exigencias actuales del mercado laboral y a las competencias requeridas, lo que facilita una búsqueda de empleabilidad más eficiente y favorece actitudes y aptitudes de emprendimiento.

» De comunicación. Se cumplen objetivos de comunicación e imagen del Centro (Facultad de Ciencias de la Comunicación de la URJC).

» Sociales. Se produce una aportación de valor a la Sociedad (conocimiento, modelos, productos, etc.) a través del uso de herramientas 2.0

» Económicos. El proyecto tiene un nulo coste añadido de los recursos para la institución, que se integra en el proceso como una empresa-cliente.

» Institucionales. Se produce una ruptura de barreras de integración y relación entre los distintos componentes de la comunidad universitaria

\section{Referencias bibliográficas}

CENTRO NACIONAL DE REFERENCIA DE APLICACIÓN DE LAS TIC BASADAS EN FUENTES ABIERTAS, 2012: "Publicaciones", en CENATIC: http://www.cenatic.es/publicaciones [Fecha de consulta: 10 de junio de 2012] 
COMISIÓN EUROPEA (2012): "Europa 2020 - La estrategia europea para el crecimiento", en Comisión Europea: http://ec.europa.eu/europe2020/index_es.htm [Fecha de consulta: 19 de octubre de 2012]

FUNDACIÓN ONCE (2012): "Publicaciones", en Editorial de la Fundación ONCE: http://www.fundaciononce.es/ES/Publicaciones/editorialFO/Paginas/Buscador.asp $\mathrm{x}$ ?TSPublicaciontitulo $=\&$ TSPublicacionautor $=\&$ TSPublicacionAnioPublicacion $=\&$ TSPublicacionclasificacion $=$ Accesibilidad [Fecha de consulta: 21 de septiembre de 2012]

GONZÁLEZ, Julia y WAGENAAR, Robert (2003): Tuning Educational Structures in Europe, Informe Fina, Fase Uno. Bilbao, Universidad de Deusto.

INTERNATIONAL ADVERTISING BUREAU (2011): "Libros blancos y estándares", en $I A B$ : http://www.iabspain.net/estandares/ [Fecha de consulta: 21 de septiembre de 2011]

ORTIZ SOBRINO, Miguel Ángel (2009): "La reconversión de la formación en comunicación desde el Espacio Europeo de Educación Superior", en Revista Icono 14 [en línea], Vol. 7, no 3. pp. 35-49. Disponible en: http://www.icono14.net . [Fecha de consulta: 6 de mayo de 2012]

PULIDO, Antonio (2003): "Los grandes cambios de futuro del sistema educativo universitario en Europa. Notas para la reflexión", en Antonio Pulido San Román Trabajos de Investigación: http://www.antoniopulido.es/documentos /con031002.pdf [fecha de consulta: 28 de septiembre de 2012]

WEB ACCESSIBILITY INITIATIVE (2011): "Strategies, guidelines, resources to make the Web accessible to people with disabilities", en WAI: http://www.w3.org/WAI/ [Fecha de consulta: 7 de julio de 2011]

\section{Sergio ÁLVAREZ GARCÍA}

Universidad Rey Juan Carlos (Campus de Fuenlabrada)

Departamento de Ciencias de la Comunicación II

Profesor Visitante Doctor. Área de Comunicación Multimedia e Interactiva sergio.alvarez@urjc.es 